\title{
Design and Analysis of MEMS Helical Antenna
}

\author{
Akshatha Arjun Babu Naidu \\ Department of Electrical and Electronics engineering \\ Kaunas University of Technology \\ Kaunas, Lithuania
}

\author{
Venkatesh Vaidhyam Kannan \\ Department of Electrical and Electronics engineering \\ Kaunas University of Technology \\ Kaunas, Lithuania
}

\begin{abstract}
Micro-Electromechanical System (MEMS) antennas in Terahertz (THz) applications has rapidly expanded in recent years due to the advent of accurate Computer Aided Design (CAD) tools. The design of a wideband MEMS helix antenna can be optimized using tree-dimensional High Frequency Structure Simulator (3D-HFSS) based on QuasiNewton (Q-N) and Sequential Non Linear Programming (SNLP) techniques to modify the antenna structure with a high accuracy for the selective band of frequencies by training the samples and minimizing the error from Finite Element Method (FEM) based simulation tool. The return loss for the helix antenna presented by MEMS technology is very low ranging from -20 to $\mathbf{- 6 5} \mathrm{dB}$ for a large range of frequencies that is from 2.5 to $5 \mathrm{THz}$. The efficiency and performance can finally be achieved by rectifying and synthesizing various tunable parameters.
\end{abstract}

Keywords-Helix antenna, MEMS, RF and Quasi-Newton optimizer.

\section{INTRODUCTION}

A. Wireless communications systems are constantly evolving towards higher data rates, longer ranges and improved robustness, together with lower costs, a lower power consumption and, in many cases, being part of compact and portable devices. Antennas, as radio-waves transceivers, play a fundamental role in this evolution. Radio frequency micro electromechanical systems (RF MEMS) are an enabling technology for a new generation of intelligent antennas with the capability of dynamically self-adapting their properties in order to maintain performance under operational or environmental variations. The tunable characteristics of MEMS can be used, for instance, to modify the antenna operation frequency or to continuously steer the antenna radiation beam towards favorable directions. [1]

From the application point of view RF MEMS antennas can provide the existing wireless systems an improved performance by exploiting their tunability properties. The improved system performances, such as data rate, range or size, are strongly linked to the different reconfigurable parameters. [2]

\section{RF MEMS ANTENNA TECHNOLOGY}

The first technological approach consists of manufacturing the antenna structure in a first step, then adding the RF MEMS devices in subsequent steps using wire-bonding and conductive epoxy. This is the main technological approach at lower frequencies, typically up to a few gigahertz. The RF MEMS devices used by these antennas are switches and variable capacitors. RF MEMS switches can be used to route the RF-signal towards different ports and to physically modify the antenna structure. These modifications lead to a low number of operation modes associated with strong variations of the antenna properties. [3]

\section{ANTENNA DESIGN AND GEOMETRY}

The geometry of the antenna and its parameters are calculated using the following formula

$0.25 \lambda_{0}<\mathrm{D}<0.45 \lambda_{\mathrm{o}}$

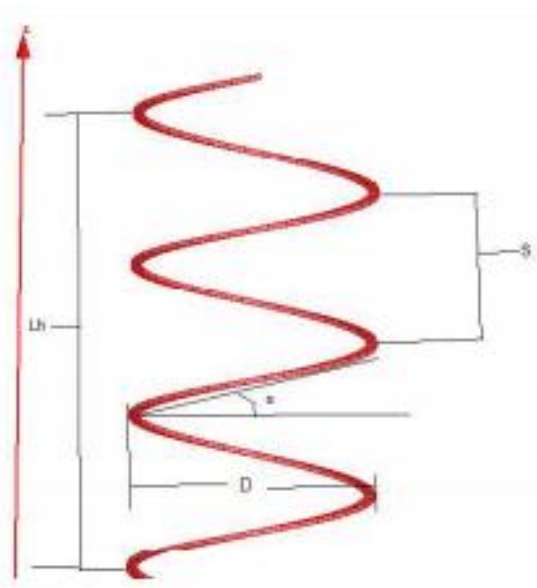

Fig. 1. 2D geometry of helix antenna [3]

Where $\mathrm{D}$ is the diameter and $\lambda$ is the wavelength of centre frequency. The fabrication is done using the process called as focused ion beam (FIB), in which the highly focused beam is used such as $\mathrm{Ga}+$ beam that scans and the cuts the substrate surface inside the vacuum chamber. This FIB process gives a great flexibility in micro scale measurements. Similarly, the $3 \mathrm{D}$ configuration is introduced for the CPW feed.

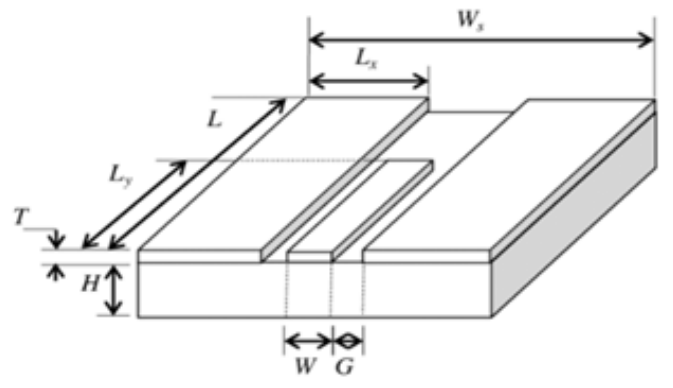

Fig. 2. CPW feed's 3D configuration [3]

In the above $3 \mathrm{D}$ configuration of a (coplanar waveguide) CPW is a single substrate of crystal silicon is fabricated employing the micromachining. Where $\mathrm{W}$ is the width of the substrate, $\mathrm{L}$ is the length of the substrate, $\mathrm{T}$ is its thickness and $\mathrm{H}$ is the height of the substrate. Here the high level of antenna 
precision is met which in turn results in large bandwidth with very low reflection coefficient. This fabrication process is highly precise and can be integrated with another device. [3]

\section{ANTENNA OPTIMIZATION AND DEVELOPMENT}

To understand the antenna optimization, it is first necessary to understand its return loss. The simulated return loss in shown below.

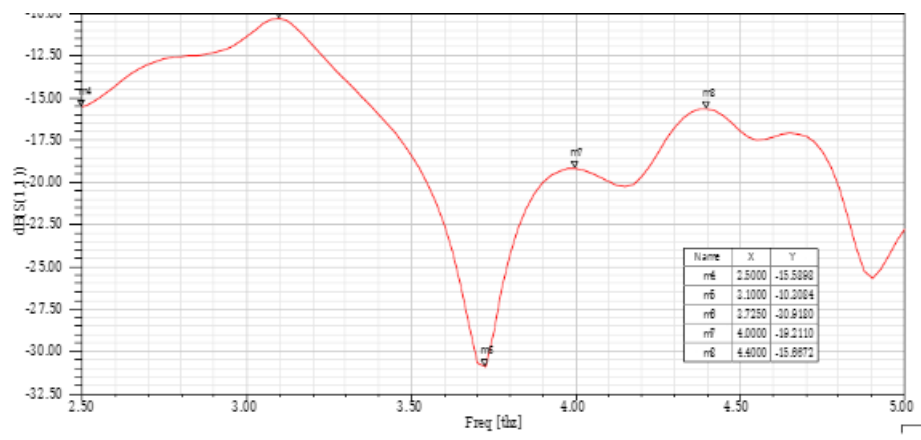

Fig. 3. Return loss of MEMS Helix antenna [4]

The above graph indicated the return loss of MEMS helix

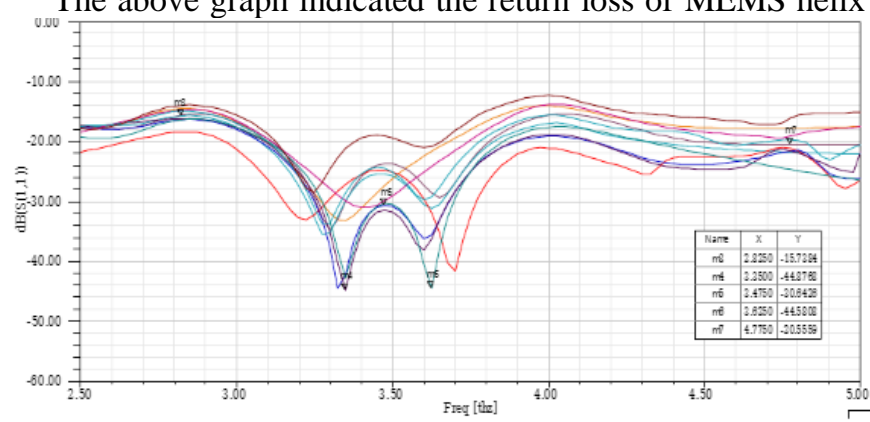

Fig. 4. Return loss of MEMS Helix antenna using Quasi-Newton optimizer. [4]

antenna configuration for the frequency ranging from 2.5 to $5.5 \mathrm{THz}$, which gives quite acceptable performance. For more precision the optimized results have to be obtained using Quasi-Newton optimization method.

Quasi-Newton optimizer is basically used to optimize three main parameters those are diameter, width, and thickness. Also, the initial point of parameter optimization is placed very close beginning variables. As an output smooth and very little numerical noise is produced. [4]

\section{RESULTS}

The whole implementation and simulation of MEMS Helical Antenna fabricated with silicon substrate with dielectric constant as 11.8 is shown below.

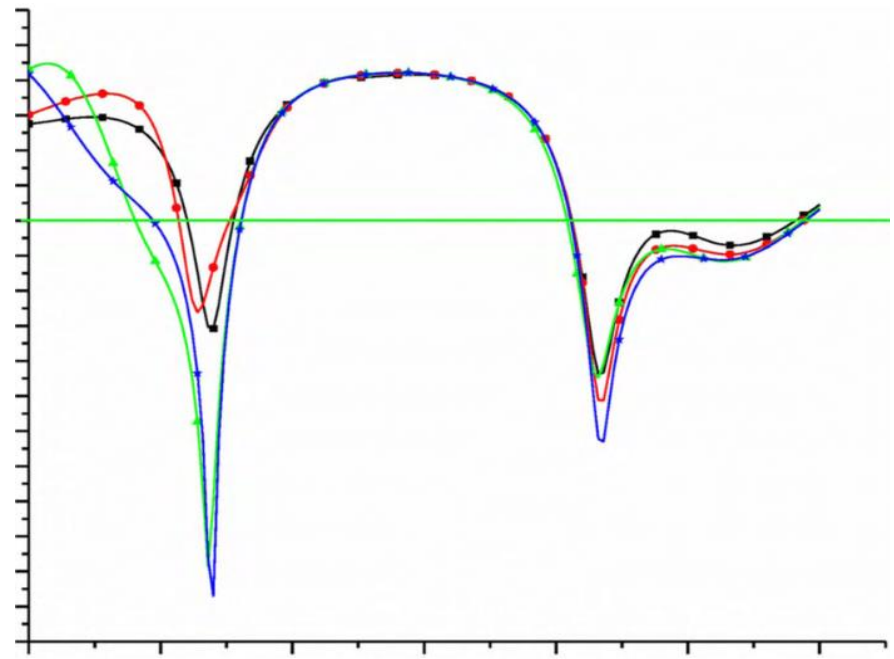

Fig. 5. Simulated return loss comparsion of MEMS Helix Antenna with and without Quasi-Netwon Optimiser. [4]

TABLE I. Optimized parameter of the helix antenna [3]

\begin{tabular}{|c|c|}
\hline Antenna Parameters & Value in $\mu \mathrm{m}$ \\
\hline D & 25 \\
\hline L & 40 \\
\hline H & 10 \\
\hline S & 15 \\
\hline W & 7.1 \\
\hline T & 1 \\
\hline N & 10 \\
\hline G & 3 \\
\hline
\end{tabular}

From the above results it could be seen that the return loss is always below $-10 \mathrm{~dB}$ within the frequency range of 2.5 to 5.5 THz. The overall substrate height value is kept constant of a $10 \mu \mathrm{m}$. and dielectric constant of 11.8. Hence when the dielectric constant values are changed the further optimization of antenna parameters occurs. In order to achieve better performance, the Newton Optimizer is added along with the helix pitch and turn number, and the CPW gap and thickness. This locates the improving points and allows fast noise and convergence reduction. Hence from the figure 7 it is clearly observed that the characterization of the MEMS Helix Antenna is improved over the entire frequency range.

\section{REFERENCES}

[1] D. Rodrigo L.Jofre J.Romeu, RF MEMS antennas for wireles applications, Handbook of Mems for Wireless and Mobile Applications.

[2] Al-Dahleh R., Shafai C., Shafai L. Frequency-agile microstrip patch antenna using a reconfigurable MEMS ground plane Microwave and Optical Technology Letters, 43 (2004), pp. 64-67

[3] Indranil Acharya, Akash Singh Chauhan and Snehanshu Sengupta, Gain enhancement of MEMS helix antenna using double substrate and fractal structures 18 June 2015 .

[4] Abdelhakim Boudkhil, Mohammed Chetioui, Nadia Benabdellah and Nasreddine Benahmed Development and Performance Enhancement of MEMS Helix Antenna for THz Applications using 3D HFSS-based Efficient Electromagnetic Optimization 